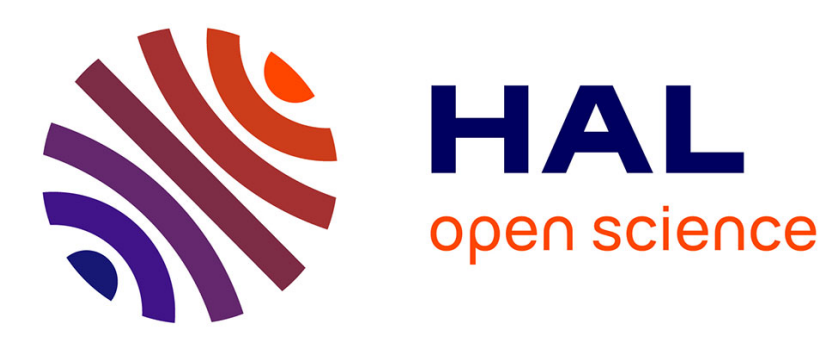

\title{
Defect-mediated turbulence in ribbons of viscoelastic Taylor-Couette flow
}

Noureddine Latrache, Nizar Abcha, Olivier Crumeyrolle, Innocent Mutabazi

\section{To cite this version:}

Noureddine Latrache, Nizar Abcha, Olivier Crumeyrolle, Innocent Mutabazi. Defect-mediated turbulence in ribbons of viscoelastic Taylor-Couette flow. Physical Review E , 2016,93 (4), pp.043126. 10.1103/PhysRevE.93.043126 . hal-01537639

\section{HAL Id: hal-01537639 \\ https://hal.science/hal-01537639}

Submitted on 12 Jun 2017

HAL is a multi-disciplinary open access archive for the deposit and dissemination of scientific research documents, whether they are published or not. The documents may come from teaching and research institutions in France or abroad, or from public or private research centers.
L'archive ouverte pluridisciplinaire HAL, est destinée au dépôt et à la diffusion de documents scientifiques de niveau recherche, publiés ou non, émanant des établissements d'enseignement et de recherche français ou étrangers, des laboratoires publics ou privés. 


\title{
Defect-mediated turbulence in ribbons of viscoelastic Taylor-Couette flow
}

\author{
Noureddine Latrache, ${ }^{1}$ Nizar Abcha, ${ }^{2}$ Olivier Crumeyrolle, ${ }^{3}$ and Innocent Mutabazi ${ }^{3}$ \\ ${ }^{1}$ Université de Brest, FRE CNRS 3744 IRDL, 29238 Brest, France \\ ${ }^{2}$ Morphodynamique Continentale et Côtière, UMR6143,CNRS-Université de Caen, Normandie Université, \\ 24, rue des Tilleuls, 14000 Caen Cedex 3, France \\ ${ }^{3}$ Laboratoire Ondes et Milieux Complexes (LOMC),UMR 6294, CNRS-Université du Havre, Normandie Université, \\ B.P. 540, 76058 Le Havre Cedex, France
}

(Received 30 April 2015; revised manuscript received 29 December 2015; published 22 April 2016)

\begin{abstract}
Transition to defect-mediated turbulence in the ribbon patterns observed in a viscoelastic Taylor-Couette flow is investigated when the rotation rate of the inner cylinder is increased while the outer cylinder is fixed. In four polymer solutions with different values of the elasticity number, the defects appear just above the onset of the ribbon pattern and trigger the appearance of disordered oscillations when the rotation rate is increased. The flow structure around the defects is determined and the statistical properties of these defects are analyzed in the framework of the complex Ginzburg-Landau equation.
\end{abstract}

DOI: 10.1103/PhysRevE.93.043126

\section{INTRODUCTION}

The addition of a small amount of polymers with high molecular mass $\left(M \sim 10^{6} \mathrm{~g} / \mathrm{mol}\right)$ to a Newtonian solvent brings viscoelastic properties to the resulting solutions. Under shear with a rate $\dot{\gamma}$, polymer solutions exhibit viscoelastic properties via the shear thinning, the relaxation time $\lambda$, and the normal stress differences that do not exist in Newtonian liquids [1]. The Taylor-Couette system that consists of a flow confined in the gap between two coaxial differentially rotating cylinders is one of the flow systems that is suitable for investigation of the viscoelastic properties of polymer solutions [2-16]. The flow in viscoelastic Taylor-Couette system with a given radius ratio or curvature can be controlled by the Taylor number $\operatorname{Ta}=\operatorname{Re}(d / a)^{1 / 2}$ for the inertia and flow curvature effects and by the elasticity number $E=\lambda / \tau_{v}$ and the viscosity ratio $S=\eta_{p} / \eta$ for the viscoelastic effects. Here $\tau_{v}$ is the viscous diffusion time, $d$ is the gap width, $a$ is the radius of the inner cylinder, $\eta$ is the solution viscosity, $\eta_{p}=\eta-\eta_{s}$ represents the polymer contribution to the solution viscosity, and $\eta_{s}$ the solvent viscosity. The elasticity number can be written as the ratio between the Reynolds number $\operatorname{Re}=\dot{\gamma} \tau_{\nu}$ and the Weissenberg number, $\mathrm{Wi}=\dot{\gamma} \lambda$, i.e., $E=\mathrm{Wi} / \mathrm{Re}$, and it characterizes the polymer solution in a flow configuration with a characteristic length $d$.

For very small values of the elasticity number, the elastic effects are very weak compared to inertia effects. In this case, experiments with different polymer solutions $[4-11,17]$ have found a stabilization of the circular Couette flow, i.e., the threshold $\mathrm{Ta}_{c}$ of the primary instability mode (Taylor Vortex Flow) is increased compared to the Newtonian case. For high values of the elasticity, $E \sim 1$, the elastic effects are very important compared to inertia effects. Pure elastic instability is observed: the circular Couette flow bifurcates to a chaotic state formed of the irregular in space and in time vortices even at very small values of the Taylor number $\mathrm{Ta}_{c}[4,7]$. For intermediate elasticity, $E \sim 10^{-2}$, the elastic and inertia effects are comparable, the circular flow bifurcates to rotating standing waves also called ribbons $[4,7,16]$, which become unstable to disordered oscillations as the control parameter Ta is increased.
Theoretical analysis of the flow solutions using the Oldroyd-B model $[12,13]$ and direct numerical simulations (DNSs) of the FENE-P model $[14,15]$ have reproduced some of the flow patterns observed in the experiments. In particular, it was shown that the ribbons bifurcate surpercritically from the base flow [12,13]. Recently Liu and Khomami [18] proposed a simple mechanism based on the comparison between elastic body force and centrifugal force to explain the inertio-elastic turbulence in the Taylor-Couette flow. Latrache et al. $[16,19]$ have shown experimentally that, for polyethylene oxide (PEO) solutions with $E \sim 0.01-0.05$, when $\mathrm{Ta}$ is increased, the ribbon pattern becomes unstable to ribbon pattern with spatio-temporal defects before the occurrence of the transient turbulent spots followed by permanent turbulent spots coexisting with laminar zones. Then this regime of spatio-temporal intermittency was replaced by the inertioelastic turbulence in which the size and the duration of the laminar zones are comparable with those of the turbulent zones. These different states (ribbon pattern with defects, transient turbulent spots, or permanent turbulent spots) belong to the disordered oscillations regime first reported in Ref. [4] in the viscoelastic Taylor-Couette flow of high molecular weight polyacrylamide solution almost two decades ago. The states with spatio-temporal defects are called defect-mediated turbulent (DMT) states [20]. To our best knowledge, no detailed investigation of the defect-mediated turbulence in viscoelastic flows has been reported so far. The present work is focused on the characterization of the spatio-temporal defects occurring in the ribbon patterns just above the supercritical bifurcation from the laminar circular Couette flow, and it supplements our previous work [16] which was centered on the spatio-temporal intermittency and inertio-elastic turbulence.

The defect mediated chaos has been observed in many experiments with Newtonian fluids (Bénard-Marangoni convection [21], Faraday waves [22,23], in Taylor-Dean flow [24], in inclined layer thermal convection [25], in the torsional Couette flow [26], in the counter-rotating Couette-Taylor flow [27]) and in experiments on electrohydrodynamic convection in nematic liquid crystals $[28,29]$. Theoretical analysis of the defects dynamics has been developed by Refs. [20,30-32] in the framework of the complex Ginzburg-Landau equation 
(CGLE) describing the space-time dynamics of the complex order parameter $A$ of the field pattern:

$$
\frac{\partial A}{\partial t}=A+\left(1+i c_{1}\right) \frac{\partial^{2} A}{\partial z^{2}}-\left(1-i c_{3}\right)|A|^{2} A,
$$

where $t$ is the time, $z$ is the space coordinate, and $c_{1}$ and $c_{3}$ represent the linear and nonlinear dispersion coefficients. This equation admits plane waves solutions that are linearly unstable to the Benjamin-Feir instability above the Newell line $1-c_{1} c_{3}=0$ in the plane $\left(c_{1}, c_{3}\right)$. Above the Newell line, different research groups working on numerical simulations of the CGLE have found spatio-temporal chaotic regimes without defects that were termed "phase turbulence" and spatio-temporal chaotic states with defects that were termed "defect-mediated turbulence" or "amplitude turbulence" [33-37]. Recent reviews on defect dynamics can also be found in Refs. [30,38].

In the present work, we will show that, in the small interval of the control parameter Ta just above the transition from the laminar circular Couette flow to the ribbon pattern, the perfect ribbon state undergoes a Benjamin-Feir instability that leads, when Ta is increased, to spatio-temporal defects associated with strong amplitude variation of the ribbon pattern. The main objective is to characterize the flow structure around a defect and the statistics of the defects observed in four polymer solutions with elasticity numbers $E \in\{0.011,0.020,0.046,0.137\}$ just above the threshold of the ribbon pattern. For each solution, the increase of Ta leads to states in which the number of defects first decreases and then increases with Ta. We have determined the number of defects that occur during the relation time and the associated statistics: the correlation time and the correlation length, the lifetime of a defect, the laminar time, and the distance between consecutive defects. The defects are the source of the both spatial and temporal disorder.

The paper is organized as follows: Sec. II describes the experimental setup and solution characterization. Results are described in Sec. III, and they are discussed in Sec. IV. Section V consists of our conclusions.

\section{EXPERIMENTAL SETUP AND POLYMER CHARACTERIZATION}

The experimental setup consists of a Couette cell with two coaxial horizontal cylinders. The inner cylinder is made of black Delrin with a radius $a=4.46 \mathrm{~cm}$, and the outer cylinder is made of Plexiglass with a radius $b=5.05 \mathrm{~cm}$. The gap size is $d=0.59 \mathrm{~cm}$ and the working length $L=$ $27.5 \mathrm{~cm}$. The radius ratio is $a / b=0.883$ and the aspect ratio is $\Gamma=L / d=46.6$. The outer cylinder is fixed, while the inner cylinder is driven by a DC servomotor at variable angular frequency $\Omega$ (the experimental control parameter). The average shear rate in the flow is defined as $\dot{\gamma}=\Omega a / d$. Polymer solutions were prepared by mixing a solution of polyethyleneoxide (PEO) of large molecular mass $(M \sim$ $10^{6} \mathrm{~g} / \mathrm{mol}$ ) with $40 \mathrm{ml}$ of isopropyl alcohol in $760 \mathrm{ml}$ of water. The experiments were performed for four solutions of PEO with concentrations $c \in\{500,600,700,1200\} \mathrm{ppm}$. The resulting solutions have sufficiently low viscosities to avoid the viscous heating-induced patterns that can occur in highviscosity polymer solutions [39]. Viscosity measurements

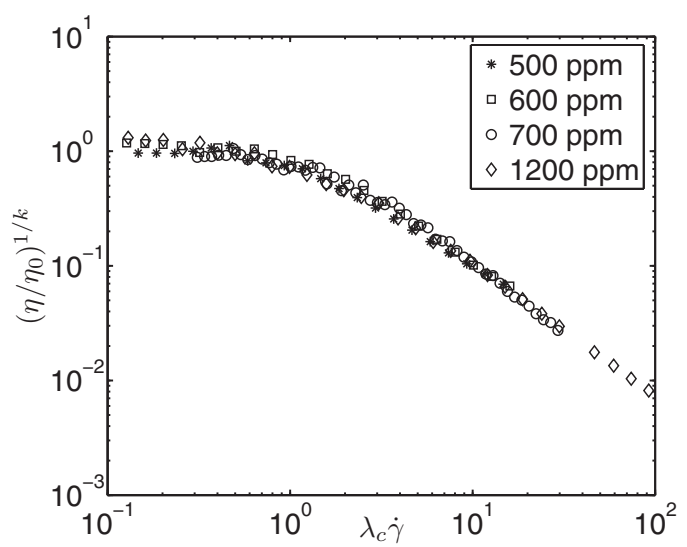

FIG. 1. Master curve of the variation of the dynamic viscosity of polymer solutions of PEO in 95\% water-5\% isopropyl alcohol with the dimensionless shear rate $\lambda_{C} \dot{\gamma}$ for three working concentrations.

revealed a shear-thinning behavior (Fig. 1), described by the Carreau viscosity formula $\eta=\eta_{0}\left\{1+\left[\lambda_{C}(\dot{\gamma})\right]^{2}\right\}^{-k}$, where $\eta_{0}$ is the plateau viscosity, $\lambda_{C}$ is the Carreau characteristic time, and $k$ is the shear-thinning index. The solution relaxation time $\lambda$ was extracted from the viscosity curve in the shear-thinning zone as the time for which the viscosity reaches $0.95 \eta_{0}$, i.e., $\lambda=\lambda_{C}\left(0.95^{-1 / k}-1\right)^{-1 / 2}$. The polymer contribution to the viscosity at the plateau (i.e., at low shear rates) is $\eta_{p 0}=\eta_{0}-\eta_{s}$. We have determined the molecular relaxation time $\lambda_{M}$ using the Flory relation $\lambda_{M}=\eta_{s} R_{g}^{3} /\left(k_{B} T\right)$ and the coupling of size-exclusion chromatography and multiangle laser light scattering to measure the gyration radius $R_{g}$ [40]. We found $R_{g}=1.6510^{-7} \mathrm{~m}$ and $\lambda_{M}=10^{-3} \mathrm{~s}$. The coefficients of Carreau formula and relaxation times of the working concentration are summarized in Table I. The two largest time scales of the flow are the solution relaxation time $\lambda$ (Table I) and the viscous diffusion time $\tau_{v}=\rho d^{2} / \eta(\dot{\gamma})$ (Table II).

For flow visualization, we have added to polymer solutions $2 \%$ of Kalliroscope AQ 1000 suspension of highly anisotropic reflective platelets. No significant change of the solution viscosity due to these platelets was observed. A linear 1024pixel CCD camera recorded the reflected light intensity $I(z)$ from a line (parallel to the cylinder axis) with 8-bit sampling. Recorded lines at regular intervals $(0.2 \mathrm{~s})$ are stacked together to make space-time diagrams $I(z, t)$ along a line of flow patterns. The duration of the data recording is $T_{\text {rec }}$ over a length $L_{\mathrm{rec}}$; in most of the experiments, $T_{\mathrm{rec}}=18618 \lambda$ and $L_{\mathrm{rec}}=$ $41 \mathrm{~d}$. In order to characterize quantitatively the hydrodynamic fields in the neighborhood of the defects, we have used the particle image velocimetry (PIV) to measure velocity fields in

TABLE I. Carreau characteristics for each polymer solutions. The solution with concentration $c=1200 \mathrm{ppm}$ was used for PIV measurements.

\begin{tabular}{lcccccc}
\hline \hline$c(\mathrm{ppm})$ & $\eta_{0}(\mathrm{mPa} \mathrm{s})$ & $\lambda_{C}(\mathrm{~s})$ & $k$ & $\lambda(s)$ & $S_{0}=\eta_{p 0} / \eta_{0}$ & $\lambda / \lambda_{M}$ \\
\hline 500 & 5.13 & 0.12 & 0.078 & 0.088 & 0.83 & 88 \\
600 & 7.40 & 0.16 & 0.098 & 0.122 & 0.88 & 122 \\
700 & 10.21 & 0.29 & 0.105 & 0.233 & 0.92 & 233 \\
1200 & 22.63 & 0.69 & 0.116 & 0.918 & 0.96 & 918 \\
\hline \hline
\end{tabular}


TABLE II. Critical values of the Weissenberg number, $\mathrm{Wi}_{c}$, the Taylor number, $\mathrm{Ta}_{c}$, for the appearance of the ribbon patterns from laminar Couette flow for each polymer solution, and their critical wave number $q_{c}$ and frequency $f_{c}$.

\begin{tabular}{lcccccccc}
\hline \hline$c(\mathrm{ppm})$ & $\eta / \eta_{0}$ & $\tau_{v}(\mathrm{~s})$ & $E$ & $S$ & $\mathrm{Wi}_{c}$ & $\mathrm{Ta}_{c}$ & $q_{c}$ & $f_{c}$ \\
\hline 500 & 0.88 & 7.8 & 0.011 & 0.809 & 1.55 & 48.6 & 3.39 & 0.014 \\
600 & 0.78 & 6 & 0.020 & 0.853 & 2.43 & 43.4 & 3.33 & 0.021 \\
700 & 0.67 & 5 & 0.046 & 0.875 & 5.24 & 41.6 & 3.29 & 0.044 \\
1200 & 0.66 & 6.7 & 0.137 & 0.961 & 11.53 & 41.9 & 2.2 & 0.15 \\
\hline \hline
\end{tabular}

meridional cross section, i.e., in the $(r, z)$ plane $[41,42]$. These measurements were performed in a vertical Tayor-Couette cell with $a=4 \mathrm{~cm}$ and $b=5 \mathrm{~cm}$ and $L=45 \mathrm{~cm}$ designed especially for PIV; it is immersed in a large rectangular tank with a temperature fixed at $T=22{ }^{\circ} \mathrm{C} \pm 0.2{ }^{\circ} \mathrm{C}$.

\section{RESULTS}

Lengths are scaled by the gap width $d$ and times are scaled by the relaxation time $\lambda$ for each concentration (Table I). For high molecular mass PEO solutions with small concentrations $c<400$ ppm corresponding to $E<0.01$ in our horizontal Taylor-Couette system, the laminar flow bifurcates to stationary Taylor vortex flow [7]. The base flow bifurcates to ribbon state for $500<c<800$ ppm (Fig. 2) and to disordered pattern for $c>800 \mathrm{ppm}$. The threshold $\mathrm{Ta}_{c}$ depends on the concentration $c$ or on the elasticity number $E$ of the polymer solution. The bifurcation to stationary Taylor vortex and to the ribbon state are supercritical, no significant hysteresis was found when increasing and decreasing Ta around the critical value $\mathrm{Ta}_{c}$ in agreement with theoretical predictions [12]. The bifurcation scenario in polymer solutions with $E<0.01$ was described in Ref. [7]; we will present the results of the experiments performed in PEO solutions with four different concentrations: $c \in\{500,600,700,1200\} \mathrm{ppm}$. In our flow systems, this range of concentrations corresponds to elasticity number $E$ from 0.011 to 0.137 where elastic and hydrodynamic stresses become comparable when $\mathrm{Ta}=\mathrm{Ta}_{c}$ and the circular Couette flow bifurcates to a standing wave also called a ribbon [Fig. 3(a)]. The ribbon state results from the superposition of left and right traveling helicoidal waves called spirals in the Taylor-Couette literature [43]. The left and right spirals have almost the same values of the frequency and of the axial wave numbers. It was found that the critical

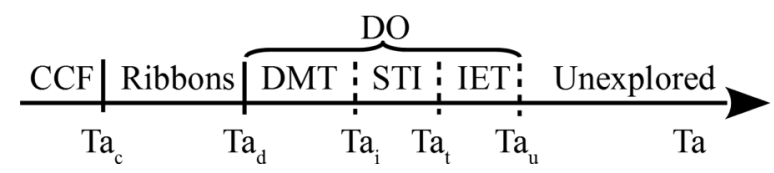

FIG. 2. Transition sequences from circular Couette flow (CCF) to the states of disordered oscillations in viscoelastic Taylor-Couette flow with concentrations $c \in\{500,600,700\}: \mathrm{Ta}_{c}, \mathrm{Ta}_{d}, \mathrm{Ta}_{i}$, and $\mathrm{Ta}_{t}$ are the threshold values of the ribbon pattern, of the defects mediated turbulence, of the spatio-temporal intermittency and of the inertioelastic turbulence [16], respectively. $\mathrm{Ta}_{u}$ is the lower boundary of the unexplored yet zone.
Taylor number $\mathrm{Ta}_{c}$ decreases with elasticity $E$ while the critical Weisenberg number $\mathrm{Wi}_{c}$ increases with $E$ and with $S$ [7]; critical values are given in Table II. The ribbon pattern was also obtained in nonlinear stability analysis of Oldroyd-B flow equations $[12,44]$, but this flow model does not contain shear thinning. We note that the ribbons appear in the shear-thinning zone where effective viscosity $\eta(\dot{\gamma})$ is lower than the viscosity of Newtonian plateau $\eta_{0}$. The main results to be analyzed are illustrated in Figs. 3, 4, and 5.

\section{A. Ribbon patterns with defects}

Increasing the angular velocity of the inner cylinder, the ribbon pattern [Fig. 3(a)] undergoes a Benjamin-Feir instability characterized by the occurrence of side-band frequency peaks located at $f_{0} \pm \Delta f$, i.e., around the fundamental peak in the frequency spectrum [Fig. 3(d)]. For $E=0.011$, the fundamental frequency of the ribbon pattern $f_{0}$ decreases as Ta increases while the axial wave number remains almost constant within the experimental precision. The ribbon pattern transits to a state of ribbons with spatio-temporal defects [Fig. 3(b)] that occur erratically in time and space. A further increase of Ta leads to the spatio-temporal intermittency (STI) regime [Figs. 2, 3(c)]. The regimes of disordered oscillations [Figs. 2, 3(b) and 3(c)] are characterized by the increase of the background noise and of the width of the peaks in the pattern spectra [Figs. 3(e) and 3(f)].

The defects are observed in the ribbon pattern just above the threshold in the interval $\left(0.02<\epsilon=\left(\mathrm{Ta}-\mathrm{Ta}_{c}\right) / \mathrm{Ta}_{c}<0.15\right.$ [Fig. 4(b)]. For $E=0.011$, they occur above $\epsilon \approx 0.02$, for $E=0.02$, they are observed above $\epsilon \approx 0.07$, and for $E=$ 0.046 , they occur above $\epsilon \approx 0.04$.

Using the complex demodulation, we have extracted the phase and amplitude of the left [Figs. 4(a) and 4(d)] and of the right spiral [Figs. 4(c) and 4(f)]. Comparison of space-time diagrams of the amplitude and phase for each spiral shows the existence of two defects of opposite charges: $v_{+}=1$ for right defect when the phase presents a positive discontinuity $(+2 \pi)$ and $\nu_{-}=-1$ for left defect when the phase presents a negative discontinuity $(-2 \pi)$. The localizations of the right and left defects are illustrated by the black and white dashed squares respectively [Figs. 4(a) and 4(c)]. In the vicinity of the right defect, the amplitude of right spiral decreases strongly and vanishes in its core, while the amplitude of the left spiral has a weak change [Figs. 4(d)—-4(f)]. The same behavior of amplitudes of the right and left spirals is observed for left defect [Figs. 4(d)-4(f)]. We observe that the defects are associated with strong amplitude variation of the ribbons [Figs. 3(b) and $4(\mathrm{~b})$ ]. In the most of cases, the defects nucleate pairwise (right and left defects) (Figs. 3-5).

\section{B. Velocity field of the ribbon patterns}

The radial and axial velocity components of the ribbon patterns without and with defects have been measured using the PIV for the solution with $E=0.137$. The space-time diagrams of the radial velocity component of the ribbons without and with defects are presented respectively in Fig. 5(a) for $\mathrm{Ta}=41.9$ and Fig. 5(d) for $\mathrm{Ta}=46$. To get more information on the behavior on ribbons and defects, we have filtered the 

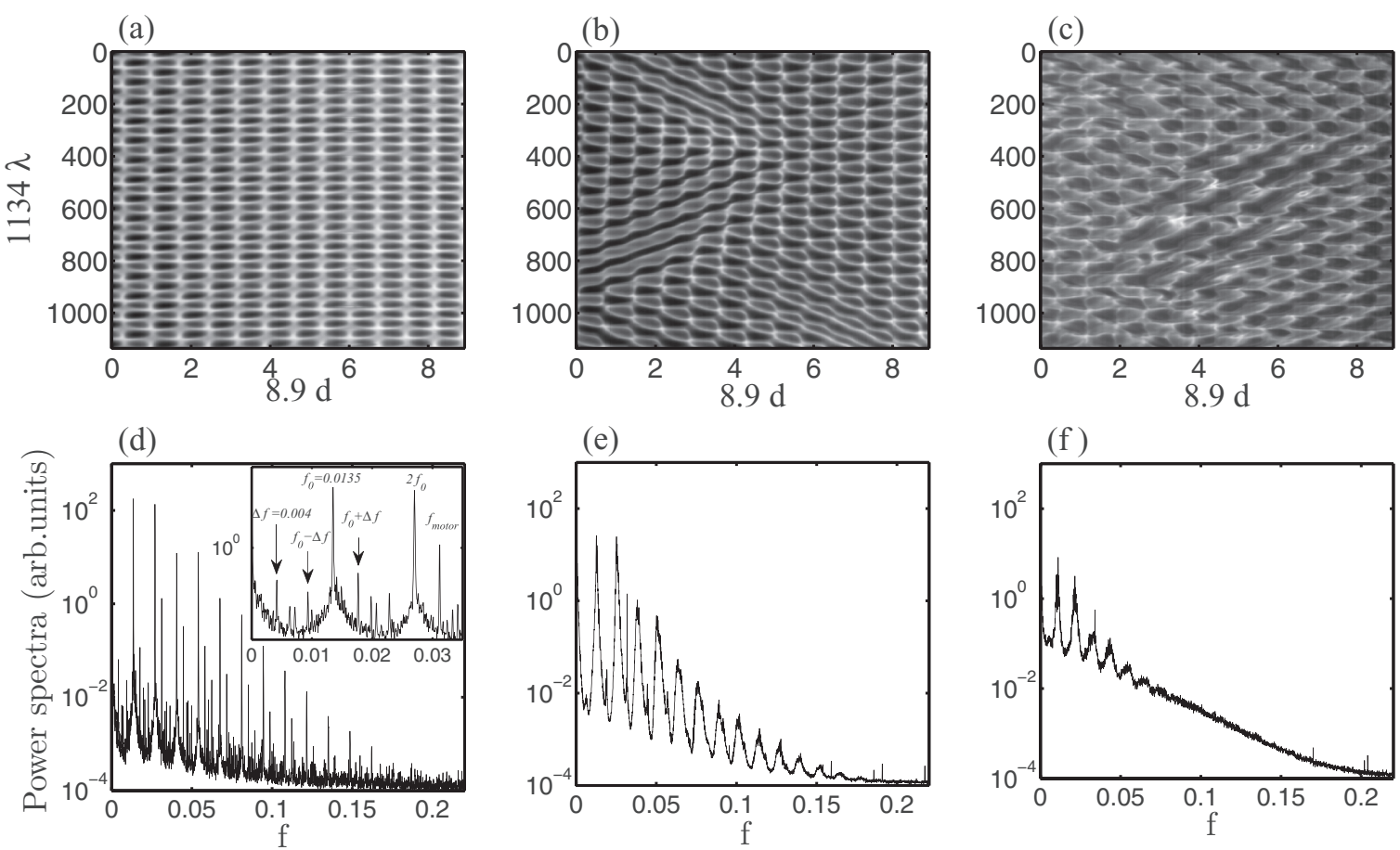

FIG. 3. Space-time diagrams and frequency power spectra of pattern states for $E=0.011$ : (a, d) ribbons without defects for Ta $=49.05$; (b, e) ribbons with defects for $\mathrm{Ta}=50.1$; (c, f) spatio-temporal intermittency regime for $\mathrm{Ta}=54.82$. The inset of Fig. 3(d) represents a zoom of power spectrum in the neighborhood of fundamental frequency.

right [Figs. 5(b) and 5(e)] and left [Figs. 5(c) and 5(f)] spirals of radial velocity components. For the ribbon state without defects, the velocity magnitudes of the right and left spirals are comparable. For the ribbons with defects [Fig. 5(d)], the velocity field of the right spiral [Fig. 5(e)] shows that the radial velocity component vanishes $\left(u_{r}^{\prime}=0\right)$ in the defects. (a)

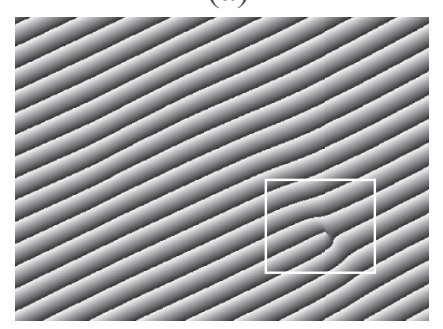

(d)

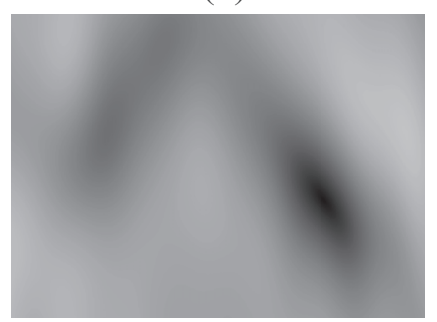

(b)

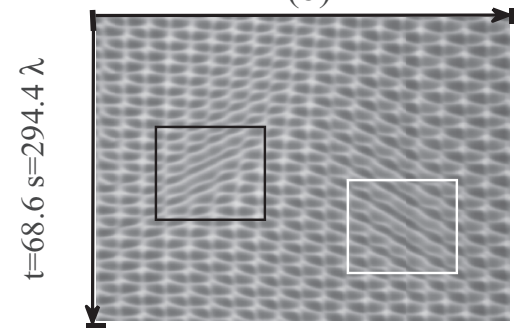

$19.4 \mathrm{~d}$

(e)

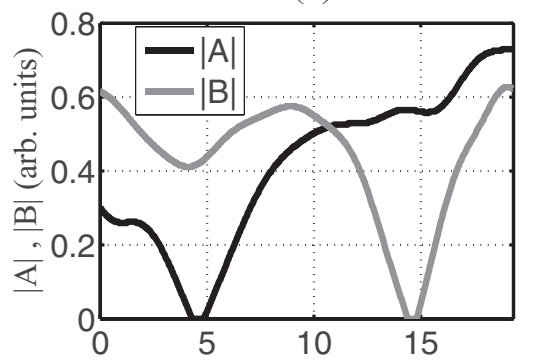

(c)

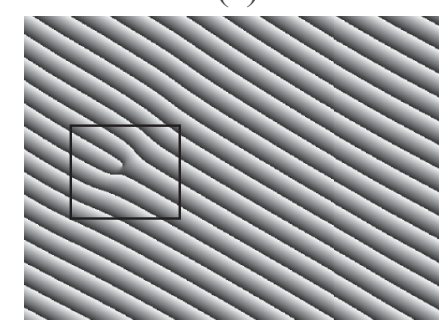

(f)

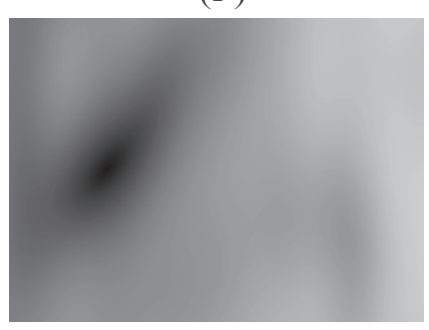

FIG. 4. Demodulation of the ribbon state observed at $\mathrm{Ta}=44.5$ and $E=0.046$ : (b) ribbons with defects the black and white dashed squares are the positions of right and left defects respectively; (a, d) phase and amplitude of the left spiral; (c, f) phase and amplitude of the right spiral; (e) spatial profiles of the amplitudes $|A|$ and $|B|$ of the right and left spirals in the vicinity of the core of defects. Black zones in amplitudes correspond to the neighborhood of the defects. 
(a)

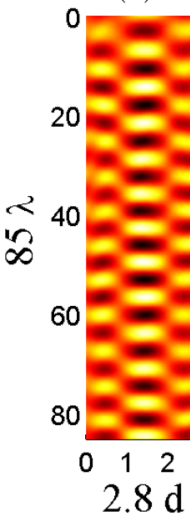

(d)

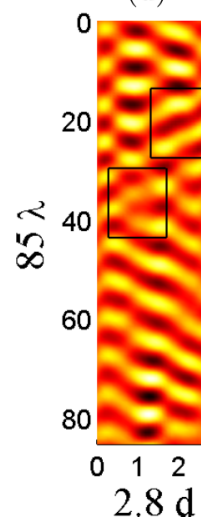

(b)

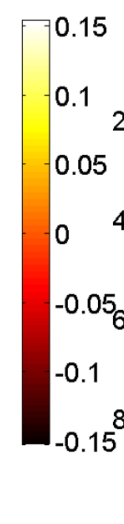

(c)

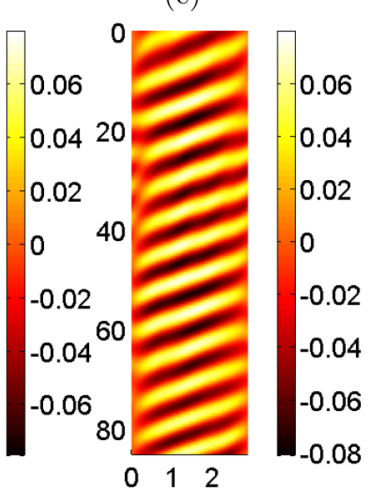

(e)

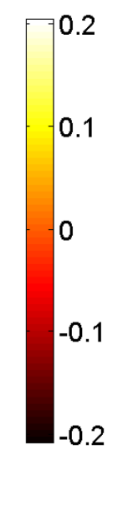

(f)

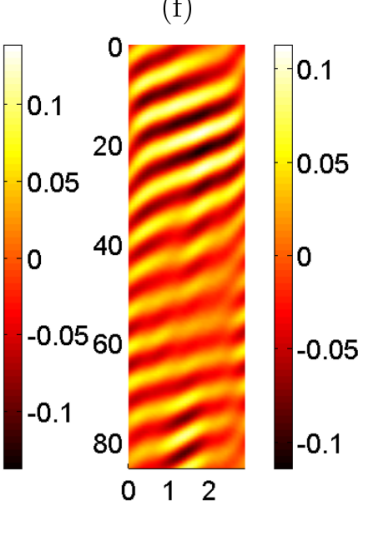

FIG. 5. Spatiotemporal diagrams of the radial velocity component for ribbon state without defects (a) for $\mathrm{Ta}=41.9$ and with defects $(\mathrm{d})$ for $\mathrm{Ta}=46$. Spatiotemporal diagrams of the the right (b, e) and left (c, f) spirals for ribbons without defects and with defects. The velocities are scaled by $d / \lambda$. The data correspond to a working solution with $E=0.137$.

We found also that the axial velocity component vanishes in the defects, i.e., $u_{z}^{\prime}=0$. The incompressibility condition implies that the azimuthal component of the perturbation also vanishes, i.e., $u_{\theta}^{\prime}=0$. These results are in a good agreement with those obtained by Refs. [27,42] in the study of the amplitude of defects in spiral pattern observed in Newtonian Couette-Taylor flow between counter-rotating cylinders and in the Taylor-Dean system [24]. They confirm the fact that the spatio-temporal defects are characterized by vanishing amplitude $A=0$ [20,29,32].

\section{Evolution of the mean number of defects with Ta}

To measure the number of defects observed in the patterns, we have digitized the space-time plot of the amplitude obtained after the complex demodulation of the space-time diagrams (Fig. 6). The dark regions in Fig. 6(b) correspond to depressions in the pattern amplitude [Fig. 6(a)] and are associated with defects. The amplitude of the ribbon pattern being zero in the core of the defects, a digitization process (black: $A=0$ for defects, white: $A>0$ for ribbon pattern) leads to the black and white pattern [Fig. 6(c)]. Then we can count the number $n$ of defects during a whole experimental
TABLE III. Fit coefficients of the defect density in different systems using the formula (3). For the present work, $c_{3}=\mathrm{Ta}$ and $c_{3}^{\prime \prime}=\mathrm{Ta}_{d}$

\begin{tabular}{lcccc}
\hline \hline System & $E$ & $a$ & $b$ & $c_{3}^{\prime \prime}$ \\
\hline $\begin{array}{l}\text { Ginzburg-Landau } \\
\text { equation [37] }\end{array}$ & 0 & 0.66 & 0.98 & 0.70 \\
$\begin{array}{l}\text { DMC in torsional } \\
\text { Couette flow [26] }\end{array}$ & 0 & 5 & 4 & 38.5 \\
Present work: & 0.011 & $0.058 \pm 0.004$ & $0.25 \pm 0.04$ & 49.8 \\
& 0.020 & $0.008 \pm 0.001$ & $0.86 \pm 0.05$ & 46.6 \\
& 0.046 & $0.032 \pm 0.001$ & $6.0 \pm 0.08$ & 43.5 \\
\hline \hline
\end{tabular}

run and determine the mean number of defects as

$$
\langle n\rangle=n / \tilde{T}_{\mathrm{rec}}=n \lambda / T_{\mathrm{rec}},
$$

where $\tilde{T}_{\text {rec }}$ is the dimensionless duration of data recoding. The variation of $\langle n\rangle$ with the control parameter is plotted in Fig. 7(a) for three polymer solutions. We observe that, for each solution (i.e., each value of the elasticity number $E$ ), there exists a value of $\mathrm{Ta}=\mathrm{Ta}_{d}$ below which the number of defects decreases and above which, the number of defects increases. For all the three working solutions, $\mathrm{Ta}_{d} / \mathrm{Ta}_{c} \in[1.025,1.075]$, meaning that the ribbon patterns become unstable to spatial modulations just after their threshold.

The value $\mathrm{Ta}_{d}$, which is a function of the elasticity number $E$ (Table III), can be considered as the threshold of the defect mediated turbulence (DMT). In fact, the dynamics of the defects observed in our experiment resembles in some aspects that observed in the transition between phase turbulence and defect turbulence of the complex GinzburgLandau equation $[33,37]$. After the analysis of the formulas suggested by different studies, we found that our data of the number of defects for $\mathrm{Ta}>\mathrm{Ta}_{d}$ are best fitted by the functional relation suggested by [37]

$$
\langle n\rangle=a \exp \left(\frac{-b}{c_{3}-c_{3}^{\prime \prime}}\right),
$$

where the nonlinear dispersion coefficient $c_{3}$ in the GinzburgLandau equation serves as a control parameter, while $a, b$, and $c_{3}^{\prime \prime}$ are free parameters. The best fit coefficients of this relation with our experimental results yields the values of $a, b$, and $c_{3}^{\prime \prime}$ given in Table III; Ta plays the same role as $c_{3}$ and $c_{3}^{\prime \prime}=\mathrm{Ta}_{d}$. The relation (3) is the analog of the Arrhenius law of the variation of the number of defects with the temperature $T$ of a crystal. The coefficient $b$ is the analog of the formation energy of a defect in the crystal, while $\mathrm{Ta}-\mathrm{Ta}_{d}$ plays the role of the thermal energy $k_{B} T$ where $k_{B}$ is the Boltzmann constant.

\section{Correlation time and correlation length}

Another way to characterize the behavior of the defects near $\mathrm{Ta}_{d}$ consists in the determination of the temporal and spatial correlation functions of the ribbon patterns without and with defects [the insets of Figs. 7(b) and 7(c) are examples of temporal and spatial correlation functions respectively] and extracted from them the correlation time and length for Ta $\epsilon$ $\left[\mathrm{Ta}_{c}, \mathrm{Ta}_{i}\right][$ Figs. $7(\mathrm{~b})$ and $7(\mathrm{c})]$. For ribbon patterns without 

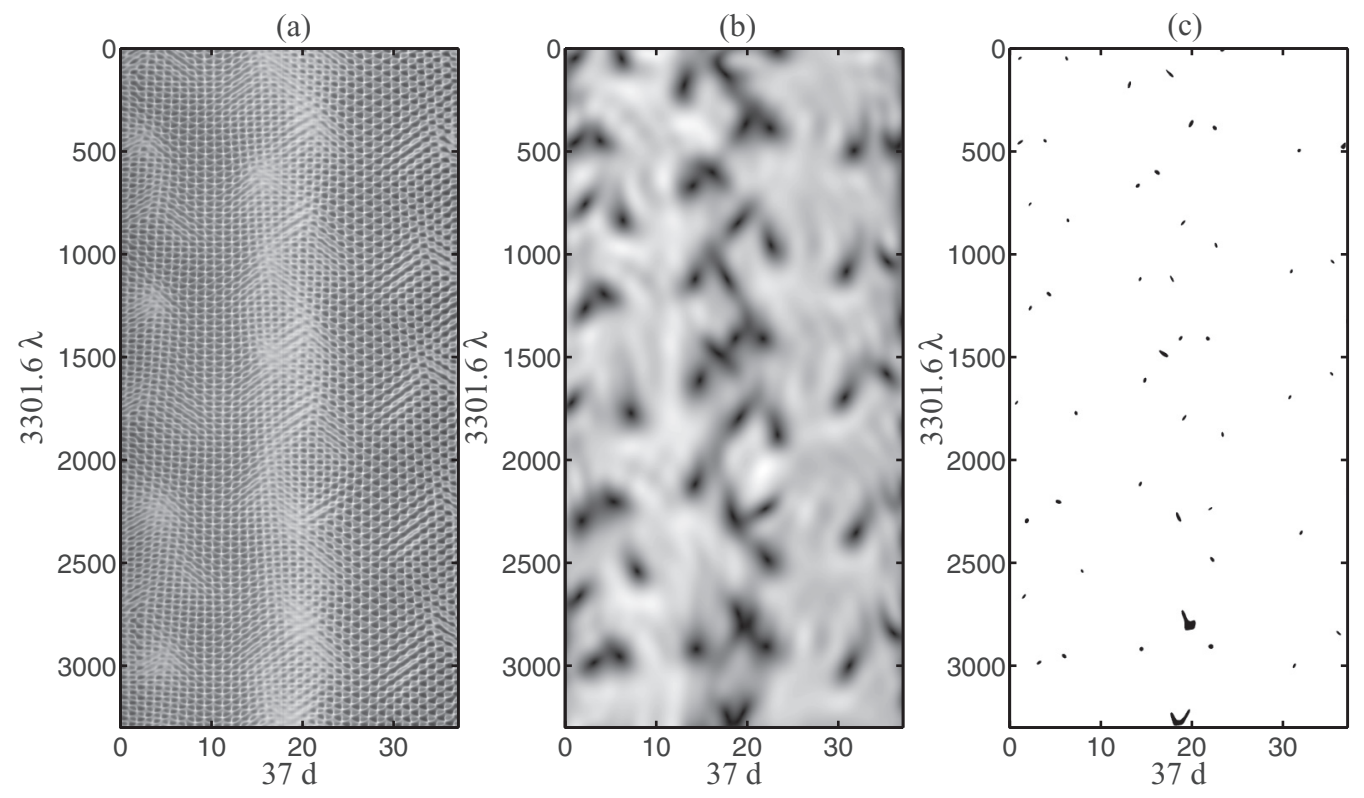

FIG. 6. Space-time diagram of: (a) ribbon pattern with defects, (b) amplitude of the pattern, (c) digitized amplitude for $E=0.020$ and $\mathrm{Ta}=46.2$.

defects, the correlation length weakly decreases (by a factor 1.2) with Ta while the correlation time decreases significantly with Ta by a factor 3 from values $\xi$ and $\tau$ at $\mathrm{Ta}_{c}$. As soon as the defects appear in the pattern, the correlation time $\tau$ drop to a small value by factor 10 and the correlation length $\xi$ is reduced by a factor 5 . In the neighborhood of the value $\mathrm{Ta}_{d}$, both $\tau$ and $\xi$ first increase for $\mathrm{Ta}<\mathrm{Ta}_{d}$ and then decrease for $\mathrm{Ta}>\mathrm{Ta}_{d}$. The data were fitted by power law in the neighborhood of $\mathrm{Ta}_{d}$ as follows:

$$
\tau \propto \begin{cases}\left(\mathrm{Ta}_{d}-\mathrm{Ta}\right)^{\gamma_{1}}, & \mathrm{Ta}<\mathrm{Ta}_{d} \\ \left(\mathrm{Ta}-\mathrm{Ta}_{d}\right)^{\gamma_{2}}, & \mathrm{Ta}>\mathrm{Ta}_{d}\end{cases}
$$

and

$$
\xi \propto \begin{cases}\left(\mathrm{Ta}_{d}-\mathrm{Ta}\right)^{\delta_{1}}, & \mathrm{Ta}<\mathrm{Ta}_{d} \\ \left(\mathrm{Ta}-\mathrm{Ta}_{d}\right)^{\delta_{2}}, & \mathrm{Ta}>\mathrm{Ta}_{d}\end{cases}
$$

The best fit coefficients $\gamma_{i}, \delta_{i}, i=1,2$ are given in Table IV. These power law fits work just in the neighborhood of $\mathrm{Ta}_{d}$. Exponential fits have been tested to capture the variation of $\tau$ and $\xi$ on the whole interval $\mathrm{Ta}_{d}<\mathrm{Ta}<\mathrm{Ta}_{i}$, but they missed most of the points near $\mathrm{Ta}_{d}$.

\section{E. Laminar and defect times in the ribbon patterns}

Figure 6(c) allows us to measure the lifetime $T_{\text {def }}^{i}$ of the defect number $i$, the time $T_{\text {lam }}$ and the axial distance

TABLE IV. Fit coefficients for the variations of the mean laminar time the correlations time and length, after the relation (7), (4), and (5) with Ta.

\begin{tabular}{lccccccccc}
\hline \hline$E$ & $\mathrm{Ta}_{d}$ & $A_{1}$ & $\beta$ & $A_{2}$ & $B$ & $\gamma_{1}$ & $\gamma_{2}$ & $\delta_{1}$ & $\delta_{2}$ \\
\hline 0.011 & 49.8 & 37 & -1.5 & 143 & -0.32 & -0.2 & -0.4 & -0.2 & -0.28 \\
0.020 & 46.6 & 49 & -0.7 & 200 & -0.16 & -0.4 & -0.46 & -0.23 & -0.23 \\
0.046 & 43.5 & 150 & -1.4 & 50 & -2.4 & -0.4 & -0.42 & -0.26 & -0.28 \\
\hline \hline
\end{tabular}

$L_{\text {lam }}$ separating the occurrence of two consecutive defects as illustrated in Fig. 8(a). The mean lifetime of defects $\left\langle T_{\text {def }}\right\rangle$ is calculated as

$$
\left\langle T_{\mathrm{def}}\right\rangle=\sum_{i=1}^{n} T_{\mathrm{def}}^{i} / n .
$$

The variation of the mean lifetime of defects with $T a$ is given in Fig. 8(b) for the three values of $E$. The mean lifetime of defects $\left\langle T_{\text {def }}\right\rangle$ is larger for solutions with small values of $E$. It is much larger than the relaxation time while it remains comparable to the diffusion time for the working solutions(i.e., $\left.\left\langle T_{\text {def }}\right\rangle \lambda / \tau_{v} \approx 1\right)$.

The mean laminar time $\left\langle T_{\text {lam }}\right\rangle$ [Fig. 8(a)] increases with Ta for $\mathrm{Ta}<\mathrm{Ta}_{d}$, while for $\mathrm{Ta}>\mathrm{Ta}_{d}$, it decreases with $\mathrm{Ta}$ [Fig. 8(c)]. The plots of $\left\langle T_{\text {lam }}\right\rangle$ exhibit a net divergence near $\mathrm{Ta}_{d}$; this suggests that $\mathrm{Ta}_{d}$ can be considered as the onset of the defect-mediated chaos. The behavior of $\left\langle T_{\mathrm{lam}}\right\rangle$ near $\mathrm{Ta}_{d}$ can be fitted by a power law for $\mathrm{Ta}<\mathrm{Ta}_{d}$ and an exponential decay for $\mathrm{Ta}>\mathrm{Ta}_{d}$ as

$$
\left\langle T_{\mathrm{lam}}\right\rangle=\left\{\begin{array}{c}
A_{1}\left(\mathrm{Ta}_{d}-\mathrm{Ta}\right)^{\beta}, \quad \mathrm{Ta}<\mathrm{Ta}_{d} \\
A_{2} \exp \left[-B /\left(\mathrm{Ta}-\mathrm{Ta}_{d}\right)\right], \quad \mathrm{Ta}>\mathrm{Ta}_{d}
\end{array} .\right.
$$

The fit coefficients $A_{1}, A_{2}, \beta$, and $B$ are given in Table IV.

We found that $\left\langle T_{\text {lam }}\right\rangle$ is comparable with $\tau$ in agreement with the results of Shraiman et al. [33]. The mean laminar distance between defects exhibit a similar divergence-like behavior as $\left\langle T_{\text {lam }}\right\rangle$ in the neighborhood of $\mathrm{Ta}_{d}$ with different power exponents.

The divergence behavior of the laminar time has been reported in the numerical simulations of the Ginzburg-Landau equation $[33,35]$ and in the experiment on torsional Couette flow [26]. As one can see from Fig. 8(c), the fluctuations of the laminar time are very strong, especially near the transition to DMT. We have found that the inverse of the mean laminar time is almost equal the mean number of defects: $\left\langle T_{\mathrm{lam}}^{-1}\right\rangle \approx\langle n\rangle$. We 


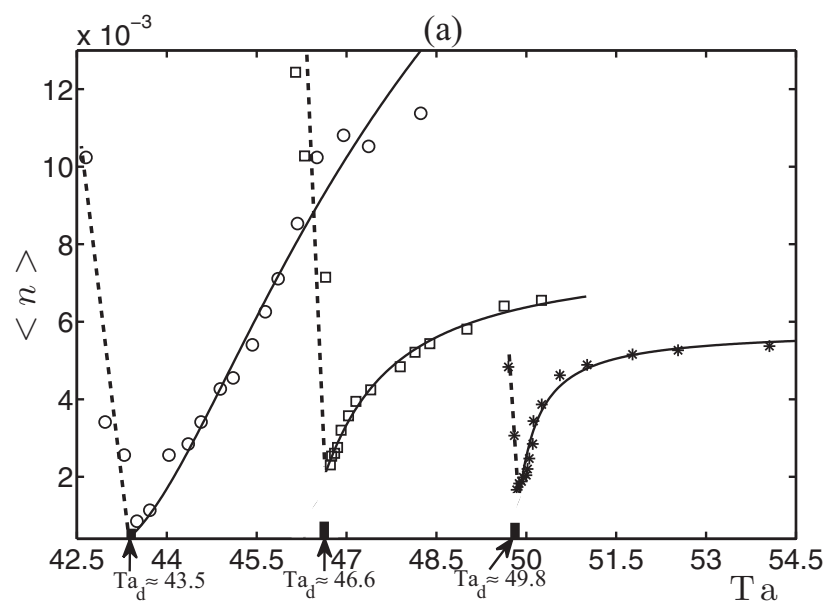

(b)

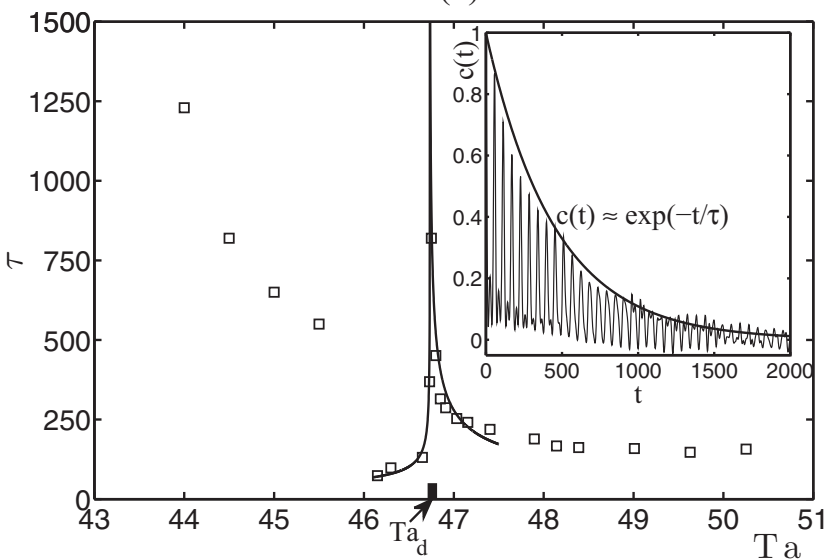

(c)

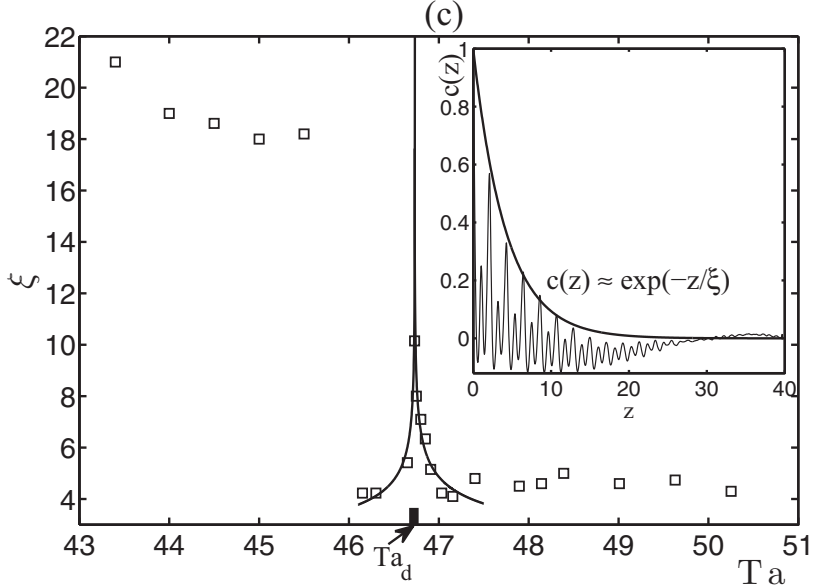

FIG. 7. Variation with Ta: (a) of the mean number of defects for three values of the elasticity number: $E=0.011$ (stars), $E=0.020$ (squares), and $E=0.046$ (circles): the solid lines are the law (3) proposed by Ref. [37]; (b) of the correlation time for $E=0.020$, (c) of the correlation length for $E=0.020$. The insets of panels (b) and (c) are examples of the temporal and spatial correlation functions, respectively. The secondary peaks in the spatial correlation function come from the subharmonic mode.

have performed a statistical analysis of laminar time between two consecutive defects for different values of Ta. Figure 9 represents an example of cumulative histogram of laminar time for $E=0.046$ and $\mathrm{Ta}=47.6$. The histograms exhibit
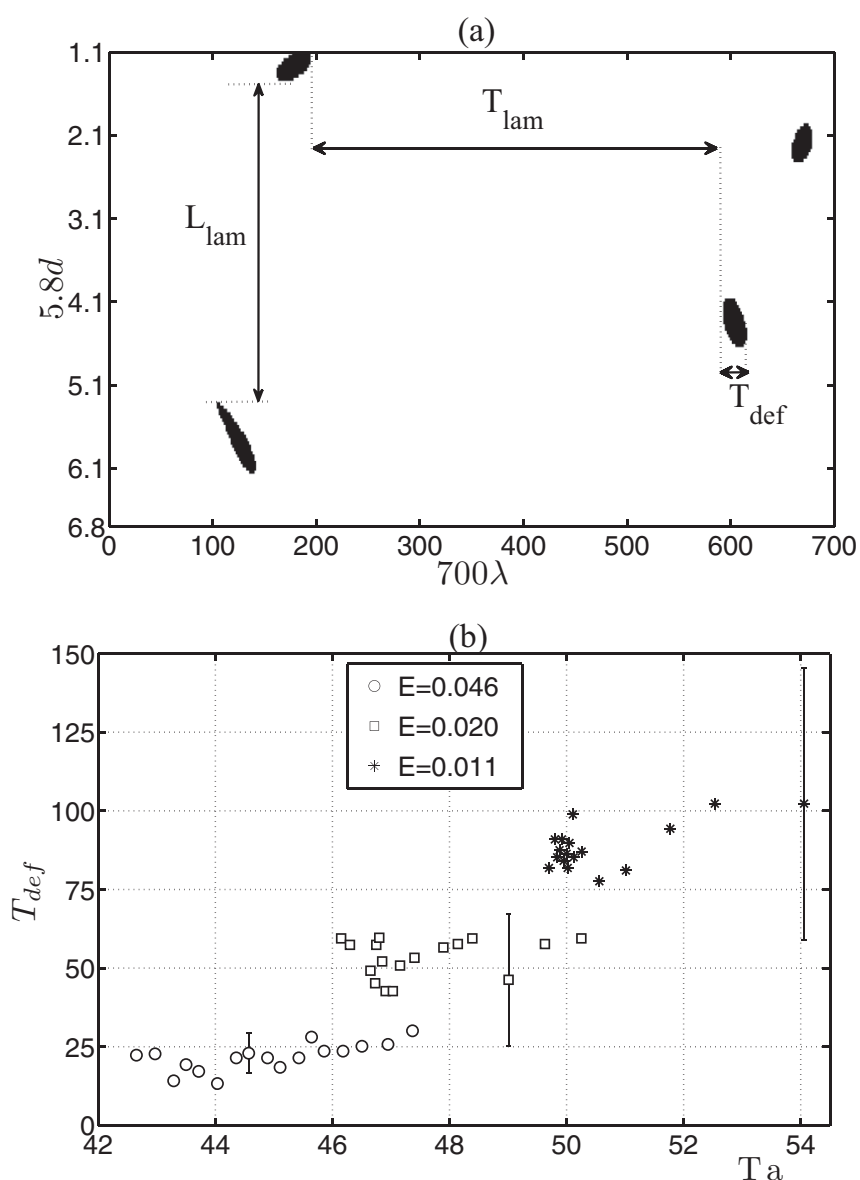

(c)

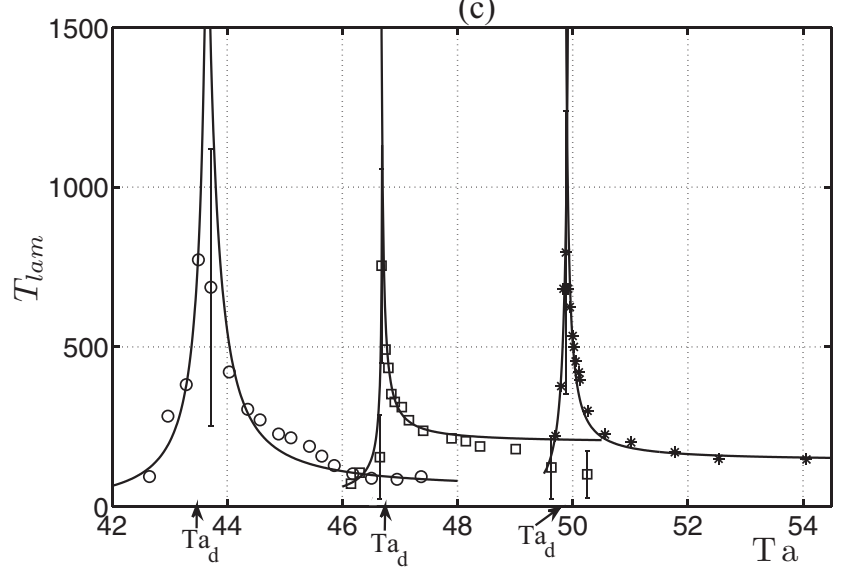

FIG. 8. (a) Digitized amplitude for $E=0.020$ and $\mathrm{Ta}=46.2$ [extracted from Fig. 6(c)]. The variation with Ta of (b) the mean lifetime of defects, (c) the laminar time between two consecutive defects, for three values of $E: E=0.011$ (stars), $E=0.020$ (squares), and $E=0.046$ (circles). The solid lines are the fits from the formula (4). The error bars represent the standard deviations.

an exponential decrease for all values of Ta and $E: N_{\text {lam }} \propto$ $\exp \left(-T_{\text {lam }} / \mathrm{Tc}_{\mathrm{lam}}\right)$, where $\mathrm{Tc}_{\text {lam }}$ is characteristic laminar time. The exponential decrease of the number of the laminar times means that the inverse of the characteristic laminar time is equal to the mean number of defects [26]. We have calculated the evolution of the inverse of the characteristic laminar time $\left(\mathrm{Tc}_{\text {lam }}^{-1}\right)$ as function as the mean number of defects $\langle n\rangle$. We 


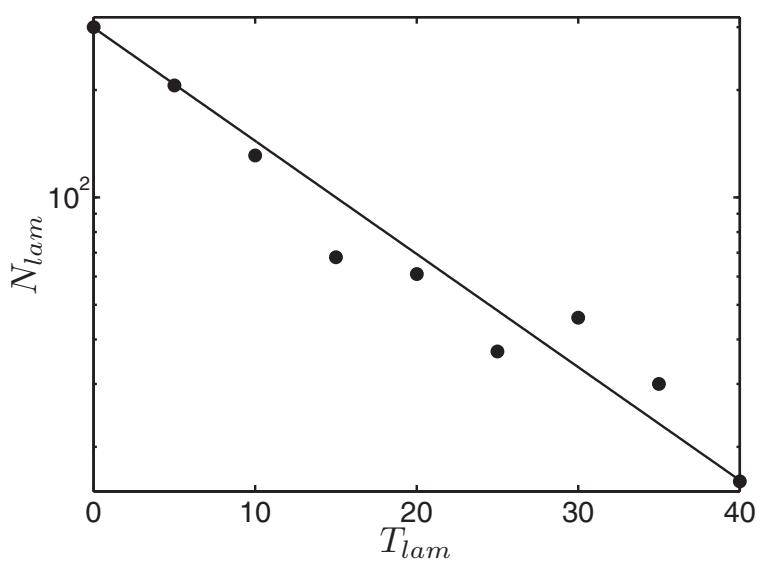

FIG. 9. Histogram of the laminar time between two consecutive defects in ribbons for $\mathrm{Ta}=47.4$ and $E=0.046$

have realized that the inverse of characteristic time is larger than the mean defect number, especially for the large values of mean number of defects, i.e., $\mathrm{Tc}_{\mathrm{lam}}^{-1}>\langle n\rangle$. This anomalous behavior can be corrected by subtracting the total cumulated duration of lifetime of defects from the acquisition duration in the calculation of the mean number of defects:

$$
\left\langle n_{\mathrm{cor}}\right\rangle=n /\left(\tilde{T}_{\mathrm{rec}}-\sum_{i=1}^{n} T_{\mathrm{def}}^{i}\right) .
$$

Figure 10 shows the evolution of the inverse of the characteristic laminar time; it is very close to the corrected mean number of defects $\left(\mathrm{Tc}_{\mathrm{lam}}^{-1} \approx\left\langle n_{\text {cor }}\right\rangle\right)$ for different values of Ta and $E$. This result confirms the one obtained by Cros and Le Gal for defect-mediated chaos in torsional Couette flow [26]. For these cases of defect-mediated chaos, the nucleation of a defect freezes the dynamics of the pattern around the defect. This result also validates the role of a homoclinic orbit in the transition scenario proposed by Afraimovich and Bunimovich [35].



FIG. 10. Variation of the characteristic laminar time with the corrected mean defect number for the three values of $E: E=0.011$ (stars), $E=0.020$ (squares), and $E=0.046$ (circles)

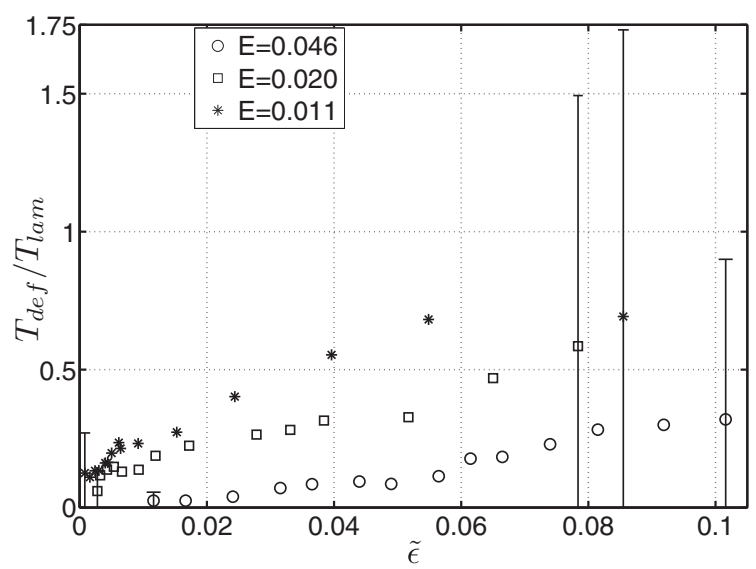

FIG. 11. Variation with $\tilde{\epsilon}$ of the ratio of the mean lifetime of defects to the mean laminar time of defects for thee three values of the elasticity number, $E=0.011$ (stars), $E=0.020$ (squares), and $E=0.046$ (circles). The error bars represent the standard deviations

\section{F. Evolution of ribbon with defects into ribbons with spots}

In order to characterize the evolution of ribbon patterns with defects into disordered ribbon pattern with spots when increasing Ta, we have compared the lifetime of defects with the laminar time between two defects. Figure 11 shows that ratio $T_{\text {def }} / T_{\text {lam }}$ increases with $\tilde{\epsilon}=\left(\mathrm{Ta}-\mathrm{Ta}_{d}\right) / \mathrm{Ta}_{d}$. The ratio $T_{\text {def }} / T_{\text {lam }}$ depends on the value of $E$ and varies between 0.3 and 0.7 when $\tilde{\epsilon} \simeq 0.1$. The mean lifetime of defect becomes comparable with the average time of turbulent spot [16]. This result confirms the theoretical prediction of Afraimovich and Bunimovitch [35] that transition occurs when the lifetime of defects is close to the laminar time $\left(T_{\mathrm{def}} \approx T_{\mathrm{lam}}\right)$ in the limit of large defect density. We have measured also the distance between two consecutive defects, and we have found that, near the threshold of the STI regime, the mean distance is close to the correlation length $\left(\left\langle L_{\text {lam }}\right\rangle \approx \xi\right)$ for the three polymer solutions, in agreement with theoretical findings of Coullet et al. [20] who showed that the transition to developed turbulence occurs when the correlation length of the system becomes of the same order of the mean distance between defects. These results confirm the fact that the defects are the precursors of the spots in ribbon patterns.

\section{DISCUSSION}

The disordered oscillations regime first observed in PAAM solutions with $M_{w}=5-6 \mathrm{MDa}$ in a Taylor-Couette system with radius ratio of 0.708 and a gap width of $0.785 \mathrm{~cm}$ and an axial aspect ratio of 54 by Ref. [4] were also observed in our Taylor-Couette system with different geometrical parameters and with different PEO solutions. These disordered oscillations can be subdivided into three states: ribbon patterns with spatio-temporal defects, ribbon patterns with transient or permanent spots and disordered state in which the laminar and turbulent spots have almost the same size (which was called inertio-elastic turbulence in Ref. [16]).

The transition from laminar viscoelastic Couette flow of ribbon state is supercritical so that the dynamics of ribbon pattern can be described by the coupled complex Ginzburg- 
Landau equations with two opposite group velocities [45]. We have shown that the defects appear on either right traveling or left traveling spirals that compose the ribbon pattern; so it is reasonable to analyze the results in the framework of the complex Ginzburg-Landau equation valid for one spiral pattern. The PIV measurements in the meridional plane of the flow have confirmed that in the ribbon state without defects, the right and left spirals have almost the same amplitude. Moreover, the amplitude of the velocity vanishes in the core of the defect in agrement with theoretical predictions.

The visualization supplemented with demodulation of the space-time diagrams of patterns has allowed us to make a statistical analysis of the defects dynamics in the disordered ribbon states. The mean number of defects, their lifetime, and the laminar time together with their correlation time and length have been measured from these diagrams, and from their behavior, a net threshold to defect-mediated turbulence in the viscoelastic Taylor-Couette flow has been determined.

The present study performed in the viscoelastic TaylorCouette flow brings a supplementary proof of the universality of the DMT in dissipative systems. In fact, the DMT was observed in different systems on the background of the oscillatory states: traveling waves in electrohydrodynamic convection in a thin layer of nematic liquid crystal [28], traveling inclined vortices in the Taylor-Dean system [24], a periodic spiral wave pattern in the torsional Couette flow [26], a state of undulations in the inclined layer convection [25], and an oscillatory transition phase in the Faraday waves [23]. This universality is described by the complex Ginzburg-Landau equation, which possesses a state diagram spanned by $\left(c_{1}, c_{3}\right)$ in which the defect states can be situated [33]. The transition to DMT in the experiments $[23,25,28]$ occurred continuously from the state without defects (the phase turbulence) to the amplitude defect turbulence by crossing the line $L_{1}$ in the plane $\left(c_{1}, c_{3}\right)$. In the present experiment, there exists defects before $\mathrm{Ta}_{d}$ whose mean number decreases towards $<n>=0$ at $\mathrm{Ta}_{d}$ as Ta increases from below, and then for $\mathrm{Ta}>\mathrm{Ta}_{d}$, the mean number of defects increases with $\mathrm{Ta}$. The correlation time and length together with the laminar time and distance between defects exhibit a divergence-like behavior near $\mathrm{Ta}_{d}$, suggesting that $\mathrm{Ta}_{d}$ represents a threshold to the defect-mediated turbulence. The existence of the defects before $\mathrm{Ta}_{d}$ whose number decreases towards zero when Ta approaches $\mathrm{Ta}_{d}$ from below can be compared to the existence of bichaotic region between lines labeled $L_{2}$ and $L_{3}$ in the plane $\left(c_{1}, c_{3}\right)$ of the CGLE. A similar behavior of the number of the defects was observed in the torsional Couette flow [26]. The existence of these defects in the present experiment and in Ref. [26] may be attributed to the length of the experiment, which is not large enough to allow the spatial modulation of the pattern to relax before generating space-time defects $[34,36]$.

\section{CONCLUSION}

In this study, we have performed velocity measurement in the meridional plane of the flow and the statistical description of the transition of the ribbon pattern to defects-mediated turbulence in viscoelastic solutions in the Taylor-Couette system when increasing the control parameter Ta for a given elasticity number $E$. The relaxation time of the viscoelastic solutions has been used to scale the temporal properties of defects. We have found that the radial and axial velocity components vanish in the core of a spatio-temporal defect. The complex demodulation of the space-time diagrams of the ribbon patterns and their digitization for defects detection have allowed us to characterize in some detail the generation of disordered oscillations observed in viscoelastic Taylor-Couette flows when the first bifurcation from laminar state leads to a ribbon pattern. The variations of the main characteristics of the spatio-temporal defects with the flow control parameters Ta and $E$ have been described and compared to the theoretical results from the complex Ginzburg-Landau equation. For each working viscoelastic solution, the curves of variation of the mean number of defects and of the correlation time and correlation length exhibit a divergence-like behavior near $\mathrm{Ta}_{d}$ corresponding to the transition to the defect mediated turbulence. The latter is one of the states that were coined "disordered oscillations" to characterize the higher modes of the ribbon pattern in viscoelastic flows in the Taylor-Couette flow [4].

\section{ACKNOWLEDGMENTS}

This work was supported partly by the CPER-HauteNormandie under the program THETE and by the FEDEREnergétique du Bâtiment. O.C. and I.M. acknowledge the financial support of the French National Research Agency (ANR) through the program Investissement d'Avenir (ANR10LABX-09-01), LABEX EMC ${ }^{3}$ (Project TUVECO).
[1] R. B. Bird, R. C. Armstrong, and O. Hassaguer, Dynamics of Polymer Liquids, Vol. 1 (Wiley, New York, 1987).

[2] R. G. Larson, E. S. G. Shaqfeh, and S. J. Muller, A purely elastic instability in Taylor-Couette flow, J. Fluid Mech. 218, 573 (1990).

[3] S. J. Muller, R. G. Larson, and E. S. G. Shaqfeh, A purely elastic transition in Taylor- Couette flow, Rheol. Acta 28, 499 (1989).

[4] A. Groisman and V. Steinberg, Couette-Taylor Flow in a Dilute Polymer Solution, Phys. Rev. Lett. 77, 1480 (1996).
[5] A. Groisman and V. Steinberg, Mechanism of elastic instability in Couette flow of polymer solutions: experiment, Phys. Fluids 10, 2451 (1998).

[6] A. Groisman and V. Steinberg, Elastic vs. inertial instability in a polymer solution flow, Europhys. Lett. 43, 165 (1998).

[7] O. Crumeyrolle, I. Mutabazi, and M. Grisel, Experimental study of inertioelastic Couette-Taylor instability modes in dilute and semidilute polymer solutions, Phys. Fluids 14, 1681 (2002).

[8] O. Crumeyrolle, N. Latrache, A. Ezersky, and I. Mutabazi, Modes dinstabilités observés dans un écoulement de CouetteTaylor viscoélastique, Mécanique Industries 4, 397 (2003). 
[9] C. S. Dutcher and S. J. Muller, The effects of drag reducing polymers on flow stability: insights from the Taylor-Couette problem, Korea-Australia Rheol. J. 21, 213 (2009).

[10] C. S. Dutcher and S. J. Muller, Effects of weak elasticity on the stability of high Reynolds number co- and counter-rotating Taylor-Couette flows, J. Rheol. 55, 1271 (2011).

[11] C. S. Dutcher and S. J. Muller, Effects of moderate elasticity on the stability of co- and counter-rotating Taylor-Couette flows, J. Rheol. 57, 791 (2013).

[12] M. Avgousti and A. N. Beris, Non-axisymmetric modes in viscoelastic Taylor-Couette flow, J. Non-Newtonian Fluid Mech. 50, 225 (1993).

[13] R. Sureshkumar, A. N. Beris, and M. Avgousti, Nonaxisymmetric subcritical bifurcations in viscoelastic TaylorCouette flow, Proc. R. Soc. London A 447, 135 (1994).

[14] D. G. Thomas, R. Sureshkumar, and B. Khomami, Pattern Formation in Taylor-Couette Flow of Dilute Polymer Solutions: Dynamical Simulations and Mechanism, Phys. Rev. Lett. 97, 054501 (2006).

[15] D. G. Thomas, B. Khomami, and R. Sureshkumar, Nonlinear dynamics of viscoelastic Taylor-Couette flow: effect of elasticity on pattern selection, molecular conformation and drag, J. Fluid Mech. 620, 353 (2009).

[16] N. Latrache, O. Crumeyrolle, and I. Mutabazi, Transition to turbulence in a flow of a shear-thinning viscoelastic solution in a Taylor-Couette cell, Phys. Rev. E 86, 056305 (2012).

[17] M. M. Denn and J. J. Roisman, Rotational stability and measurement of normal stress functions in dilute polymer solutions, AIChE J. 15, 454 (1969).

[18] N. Liu and B. Khomami, Elastically induced turbulence in Taylor-Couette flow: direct numerical simulation and mechanistic insight, J. Fluid Mech. 737, R4 (2013).

[19] N. Latrache, O. Crumeyrolle, N. Abcha, and I. Mutabazi, Destabilization of inertio-elastic mode via spatiotemporal intermittency in a Couette-Taylor viscoelastic flow, J. Phys. Conf. Ser. 137, 012022 (2008).

[20] P. Coullet, L. Gil, and J. Lega, Defect-Mediated Turbulence, Phys. Rev. Lett. 62, 1619 (1989).

[21] P. Cerisier, R. Occelli, C. Perez-Garcia, and C. Jamond, Structural disorder in Bénard-Marangoni convection, J. Phys. 48, 569 (1987).

[22] A. Kudrolli and J. P. Gollub, Localized spatio-temporal chaos in surface waves, Phys. Rev. E 54, R1052 (1996).

[23] I. Shani, G. Cohen, and J. Fineberg, Localized Instability on the Route to Disorder in Faraday Waves, Phys. Rev. Lett. 104, 184507 (2010).

[24] P. Bot and I. Mutabazi, Dynamics of spatio-temporal defects in the Taylor-Dean system, Eur. Phys. J. B 13, 141 (2000).

[25] K. E. Daniels and E. Bodenschatz, Defect Turbulence in Inclined Layer Convection, Phys. Rev. Lett. 88, 034501 (2002).

[26] A. Cros and P. Le Gal, Defect turbulence in a spiral wave pattern in the torsional Couette flow, Phys. Rev. E 70, 016309 (2004).

[27] A. B. Ezersky, N. Abcha, and I. Mutabazi, The structure of spatio-temporal defects in a spiral pattern in the Couette-Taylor flow, Phys. Lett. A 374, 3297 (2010).
[28] I. Rehberg, S. Rasenat, and V. Steinberg, Traveling Waves and Defect-Initiated Turbulence in Electroconvecting Nematics, Phys. Rev. Lett. 62, 756 (1989).

[29] G. Goren, I. Porcaccia, S. Rasenat, and V. Steinberg, Interactions and Dynamics of Topological Defects: Theory and Experimetns Near the Onset of Weak Turbulence, Phys. Rev. Lett. 63, 1237 (1989).

[30] M. van Hecke, C. Storm, and W. van Saarloos, Sources, sinks and wavenumber selection in coupled CGL equations and experimental implications for counter-propagating wave systems, Physica D 134, 1 (1999).

[31] L. Brusch, M. G. Zimmermann, M. van Hecke, M. Bär, and A. Torcini, Modulated Amplitude Waves and the Transition from Phase to Defect Chaos, Phys. Rev. Lett. 85, 86 (2000).

[32] L. Nana, A. B. Ezersky, and I. Mutabazi, Secondary structures in a one-dimensional complex Ginzburg-Landau equation with homogeneous boundary conditions, Proc. R. Soc. A 465, 2251 (2009).

[33] B. Shraiman, A. Pumir, W. Van Saarloos, P. Hohenberg, H. Chaté, and M. Holen, Spatiotemporal chaos in the onedimensional complex Ginzburg-Landau equation, Physica D 57, 241 (1992).

[34] H. Chaté, Spatiotemporal intermittency regimes of the onedimensional complex Ginzburg-Landau equation, Nonlinearity 7, 185 (1994).

[35] V. S. Afraimovich and L. A. Bunimovich, Density of defects and spatial entropy in extended systems, Physica D 80, 277 (1995).

[36] P. Manneville and H. Chaté, Phase turbulence in the twodimensional complex Ginzburg-Landau equation, Physica D 96, 30 (1996).

[37] D. A. Egolf and H. S. Greenside, Characterization of the Transition from Defect- to Phase-Turbulence, Phys. Rev. Lett. 74, 1751 (1995).

[38] I. S. Aranson and L. Kramer, The world of the complex Ginzburg-Landau equation, Rev. Mod. Phys. 74, 99 (2002).

[39] U. A. Al-Mubaiyedh, R. Sureshkumar, and B. Khomami, Influence of energetics on the stability of viscoelastic TaylorCouette flow, Phys. Fluids 11, 3217 (1999).

[40] M. Doi and S. F. Edwards, The Theory of Polymer Dynamics, Oxford Science Publications (Oxford University Press, Oxford, 1994).

[41] N. Abcha, N. Latrache, F. Dumouchel, and I. Mutabazi, Qualitative relation between reflected light intensity by Kalliroscope flakes and velocity field in the Couette-Taylor flow system, Exp. Fluids 45, 85 (2008).

[42] I. Mutabazi, N. Abcha, O. Crumeyrolle, and A. Ezersky, Application of the particle image velocimetry to the CouetteTaylor flow, in The PIV Characteristics, Limits and Possible Applications, edited by G. Cavazzini (InTech, Rijeka, 2012), Chap. 7, pp. 177-202.

[43] P. Chossat and G. Iooss, The Couette-Taylor Problem (Springer, New York, 1994).

[44] Y. L. Joo and E. S. G. Shaqfeh, The effects of inertia on the viscoelastic Dean and Taylor-Couettte flow instabilities with applications to coating flows, Phys. Fluids A 4, 2415 (1992).

[45] M. C. Cross and P. C. Hohenberg, Pattern formation outside equilibrium, Rev. Mod. Phys. 65, 851 (1993). 